\title{
Time in the mind: Using space to think about time
}

\author{
Daniel Casasanto *, Lera Boroditsky \\ Department of Psychology, Jordan Hall, Bldg. 420, Stanford University, Stanford, CA 94305, USA
}

Received 16 September 2006; revised 5 March 2007; accepted 10 March 2007

\begin{abstract}
How do we construct abstract ideas like justice, mathematics, or time-travel? In this paper we investigate whether mental representations that result from physical experience underlie people's more abstract mental representations, using the domains of space and time as a testbed. People often talk about time using spatial language (e.g., a long vacation, a short concert). Do people also think about time using spatial representations, even when they are not using language? Results of six psychophysical experiments revealed that people are unable to ignore irrelevant spatial information when making judgments about duration, but not the converse. This pattern, which is predicted by the asymmetry between space and time in linguistic metaphors, was demonstrated here in tasks that do not involve any linguistic stimuli or responses. These findings provide evidence that the metaphorical relationship between space and time observed in language also exists in our more basic representations of distance and duration. Results suggest that our mental representations of things we can never see or touch may be built, in part, out of representations of physical experiences in perception and motor action. (c) 2007 Elsevier B.V. All rights reserved.
\end{abstract}

Keywords: Metaphor; Time; Space; Embodied cognition

\footnotetext{
* Corresponding author. Tel.: +1 6173680792.

E-mail address: casasan@stanford.edu (D. Casasanto).
} 


\section{Introduction}

How do we mentally represent things that we have never experienced through the senses, like justice, mathematics, or time-travel? One possibility is that sensory and motor representations that result from physical interactions with the world are recycled to support our thinking about abstract entities. Evidence for this view has come from patterns observed in human languages. When speaking about abstract domains, people often recruit metaphors from more concrete or perceptually rich domains (Clark, 1973; Gruber, 1965; Jackendoff, 1983; Lakoff \& Johnson, 1980; Pinker, 1989; Talmy, 1988).

For example, people often talk about time using spatial metaphors (e.g., a long vacation, a short concert) (Alverson, 1994; Clark, 1973; Traugott, 1978). Aspects of time are often said to be more 'abstract' than their spatial analogues because we can perceive the spatial, but we can only imagine the temporal (Ornstein, 1969; cf., Evans, 2004). Compare the following scenarios:

(a) They moved the truck forward two meters.

(b) They moved the meeting forward two hours.

The truck in sentence (a) is a physical object that can travel through space, and whose motion we might see, hear, or feel. By contrast, in sentence (b) there is no way to experience the meeting's 'motion' through time via the senses.'

Importantly, the relationship between space and time in language is asymmetrical: people talk about time in terms of space more often than they talk about space in terms of time (Lakoff \& Johnson, 1980, 1999). This pattern in language suggests that our conceptions of space and time might be asymmetrically dependent: we construct representations of time by co-opting mental representations of space, but not necessarily the converse. Patterns in historical language change (Sweetser, 1991) and language acquisition by children (e.g., Bowerman, 1983; Clark, 1973) likewise support the idea that spatial representations are primary, and are later co-opted for other uses such as time. Evidence from psycholinguistic experiments has also provided support for this view, showing that people construct spatial representations on-line when processing statements about time (Boroditsky, 2000, 2001; Boroditsky \& Ramscar, 2002; Núñez \& Sweetser, 2006; Piaget, 1927/1969; Torralbo, Santiago, \& Lupiáñez, 2006; Tversky, Kugelmass, \& Winter, 1991), but not necessarily the reverse (Boroditsky, 2000).

In this paper we ask whether this asymmetric relationship between space and time is limited to patterns in language and language processing, or whether it extends beyond the domain of language. Is the way we think about time dependent on space even when we're not using language at all? Previous research on the experience of

\footnotetext{
${ }^{1}$ Temporal representations are often more abstract than their spatial analogues, as this example illustrates. However, some of our spatial representations may be quite abstract, as well. For example, our conception of the Milky Way galaxy's breadth is no more grounded in direct experience than our conception of its age.
} 
distance and duration has shown that the two are not independent (Benussi, 1913; Bill \& Teft, 1969; Cohen, 1967; Cohen, Hansel, \& Sylvester, 1954; Collyer, 1977; Helson, 1930; Jones \& Huang, 1982; Price-Williams, 1954; Sarrazin, Giraudo, Pailhous, \& Bootsma, 2004), but little is known about whether the relationship between the two domains is asymmetrical, in the way that has been observed in language. The purpose of the present study is to test whether the asymmetrical dependence of time on space exists even at a more basic level of the human conceptual system.

This paper describes six psychophysical experiments that tested the separability of distance and duration in human judgments. All stimuli and responses were non-linguistic. In each task, participants viewed lines or dots on a computer screen, and reproduced either their duration or their spatial displacement. Durations and displacements were fully crossed, so there was no correlation between the temporal and spatial components of the stimuli. As such, one stimulus dimension served as a distractor for the other: an irrelevant piece of information that could potentially interfere with task performance. Patterns of cross-dimensional interference were analyzed to reveal relationships between spatial and temporal representations. We reasoned that if spatial and temporal representations are symmetrically dependent on one another, then any cross-dimensional interference should be approximately symmetric: distance should modulate duration estimates, and vice versa. Alternatively, if spatial and temporal representations are independent, there should be no significant cross-dimensional interference. However, if mental representations of time are asymmetrically dependent on mental representations of space as suggested by patterns in language, then we should observe an asymmetrical pattern of cross-dimensional interference: distance should affect duration estimates more than duration affects distance estimates.

\section{Experiment 1: Growing lines}

\subsection{Methods}

\subsubsection{Participants}

Nine participants from the MIT community performed Experiment 1, in exchange for payment. ${ }^{2}$ All participants gave informed consent, and all were native monolingual speakers of English according to a language background questionnaire (i.e.,

\footnotetext{
${ }^{2}$ A total of 72 subjects from the MIT community participated in Experiments 1-6, in exchange for payment. Of these, 16 participants were removed from the analyses reported here for performing the experiment incorrectly (e.g., estimating distance when they were instructed to estimate duration), or for excessively poor performance: for each participant, duration estimates were plotted as a function of actual stimulus duration, and distance estimates were plotted as a function of actual stimulus displacement. Participants were excluded if the slope of their duration or distance estimates was less than 0.5 , as such poor performance (e.g., indicating that the 5-s lines lasted less than $2.5 \mathrm{~s}$ ) was believed to result from impatience with the repetitive task, rather than genuine inaccuracy.
} 
English was the only language they learned before age 5, and was their strongest language at time of test).

\subsubsection{Materials}

Lines of varying lengths were presented on a computer monitor (resolution $=1024 \times 768$ pixels), for varying durations. Durations ranged from 1000 to $5000 \mathrm{~ms}$ in $500 \mathrm{~ms}$ increments. Displacements ranged from 200 to 800 pixels in 75 pixel increments. Nine durations were fully crossed with nine displacements to produce 81 distinct line types. Lines 'grew' horizontally across the screen one pixel at a time, from left to right, along the vertical midline. Lines started growing 112 pixels from the left edge of the monitor on average, but the starting point of each line was jittered with respect to the average starting point ( $+/-$ up to 50 pixels), so that the monitor would not provide a reliable spatial frame of reference. Each line remained on the screen until it reached its maximum displacement, and then it disappeared.

\subsubsection{Procedure}

Participants viewed 162 growing lines, one line at a time, from a viewing distance of approximately $50 \mathrm{~cm}$. The word "ready" appeared in the center of an otherwise blank screen for two seconds immediately before each line was shown. Immediately after each line was shown, a prompt appeared in either the upper left or lower left corner of the screen indicating that the subject should reproduce either the line's displacement (if an ' $\mathrm{X}$ ' icon appeared), or its duration (if an 'hourglass' icon appeared). To estimate displacement, subjects clicked the mouse once on the center of the X, moved the mouse to the right in a straight line, and clicked the mouse a second time to indicate that they had moved a distance equal to the maximum displacement of the stimulus. Whereas stimuli grew from a jittered starting point on the vertical midline of the screen, responses were initiated at a fixed starting point in either the upper or lower left corner. Thus, the response was translated both vertically and horizontally with respect to the stimulus. To estimate duration, subjects clicked the mouse once on the center of the hourglass icon, waited the appropriate amount of time, and clicked again in the same spot.

All responses were self-paced. For a given trial, subjects reproduced either the displacement or the duration of the stimulus, never both. Response data were collected for both the trial-relevant and the trial-irrelevant stimulus dimensions, to ensure that subjects were following instructions.

\subsection{Results and discussion}

Results of Experiment 1 showed that spatial displacement affected estimates of duration $\left(y=0.63 x+2503, r^{2}=.94, \mathrm{df}=7, p<.001\right)$, but duration did not affect estimates of spatial displacement $\left(y=0.003 x+440, r^{2}=.05, \mathrm{df}=7\right.$, ns; Figs. 1a and $\mathrm{b}, 2 \mathrm{a})$. For stimuli of the same average duration, lines that traveled a shorter distance were judged to take a shorter time, and lines that traveled a longer distance were judged to take a longer time. Subjects incorporated irrelevant spatial information in their temporal estimates, but not vice versa. This behavioral asymmetry was 

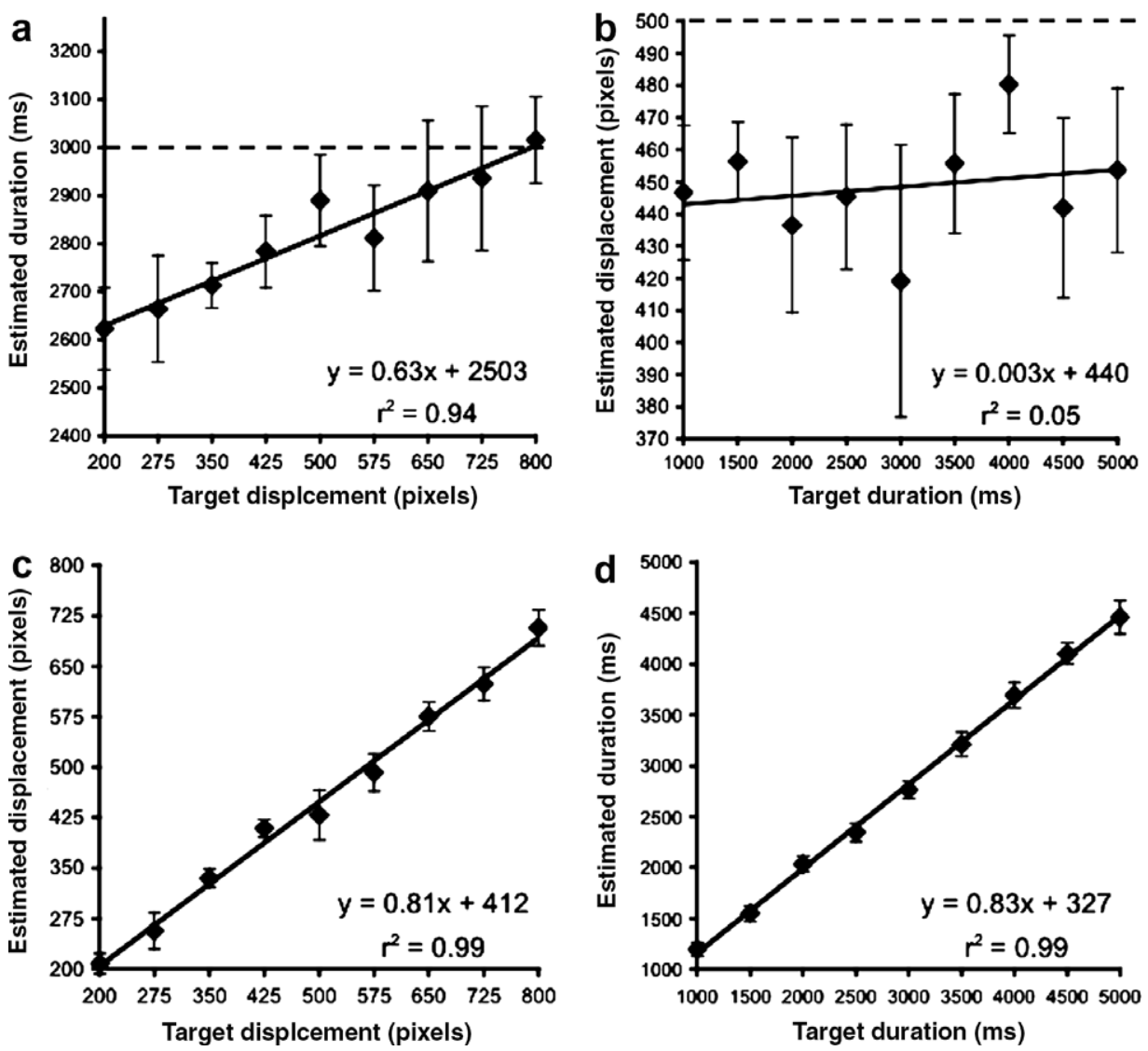

Fig. 1. Grand averaged duration and displacement estimates for Experiment 1. Top: cross-domain effects. (a) left: effect of actual line displacement on estimated duration. (b) right: effect of actual line duration on estimated displacement. The horizontal dotted lines indicate perfect performance (i.e., because target displacements and durations were fully crossed, for each actual displacement the average of all actual durations was $3000 \mathrm{~ms}$, and for each actual duration the average of all actual displacements was 500 pixels). The ranges of the ordinates of (a) and (b) are proportionate with respect to the total range of actual durations and displacements. Bottom: within-domain effects. (c) left: effect of actual line displacement on estimated displacement. (d) right: effect of target duration on estimated duration. Error bars indicate SEM.

predicted based on the asymmetrical relationship between time and space in linguistic metaphors.

Overall, estimates of duration and displacement were highly accurate, and about equally accurate in the two domains (effect of target displacement on estimated displacement: $y=.081 x+412, r^{2}=.99, \mathrm{df}=7, p<.001$; effect of target duration on estimated duration: $y=.83 x+327, r^{2}=.99, \mathrm{df}=7, p<.001$. Figs. $1 \mathrm{c}$ and $\mathrm{d}$ ). The asymmetrical cross-dimensional interference we observe cannot be attributed to a difference in the overall accuracy of duration and displacement estimations, as no significant difference was found $\left(r_{\text {duration }}-r_{\text {displacement }}=0.00, z=0.00, \mathrm{~ns}\right)$. 


\section{Experiment 2: Growing lines, selective attention}

In the first experiment, participants did not know until after each line was presented whether they would need to estimate displacement or duration. They had to attend to both the spatial and temporal dimensions of the stimulus. Experiment 2 addressed the possibility that cross-dimensional interference would diminish if participants were given the opportunity to attend selectively to the trial-relevant stimulus dimension, and to ignore the trial-irrelevant dimension.

\subsection{Materials and procedure}

Nine participants from the MIT community performed Experiment 2, in exchange for payment. Stimulus materials were identical to those used in Experiment 1. The procedure was also identical, with one exception. In Experiment 1, the word "ready" appeared for two seconds immediately preceding each line stimulus. In Experiment 2 (and all subsequent experiments reported here), the word "ready" was replaced either by the word "Space" next to an ' $\mathrm{X}$ ' icon, or by the word "Time" next to an hourglass icon. These words and symbols indicated whether the subject would need to estimate the displacement or the duration of the next line. Line stimuli, prompts, and responses were exactly as in Experiment 1, thus all stimuli and responses remained entirely non-linguistic.

\subsection{Results and discussion}

Results of Experiment 2 replicated those of Experiment 1 (cross-domain effects: effect of displacement on duration estimation: $y=0.74 x+2474, r^{2}=.92$, $\mathrm{df}=7$, $p<.001$; effect of duration on displacement estimation: $y=0.003 x+464, r^{2}=.09$, $\mathrm{df}=7$, ns. Within-domain effects: Effect of target displacement on estimated displacement: $y=0.85 x+49, r^{2}=.99, \mathrm{df}=7, p<.001$; effect of target duration on estimated duration: $\left.y=0.77 x+526, r^{2}=.99, \mathrm{df}=7, p<.001\right)$. Participants were able to disregard line duration when estimating displacement. By contrast, they were unable to ignore line displacement, even when they were encouraged to selectively attend to duration (Fig. 2b). The cross-dimensional effect of space on time estimation in Experiment 1 was not caused by a task-specific demand for subjects to encode spatial and temporal information simultaneously.

Response data collected for the trial-irrelevant dimension confirmed that participants understood the task, and were not explicitly confusing displacement with duration (i.e., participants were not giving a spatial response when they were supposed to give a temporal response).

\section{Experiment 3: Growing lines, temporal frame of reference}

Experiments 3-5 addressed concerns that spatial information in the stimulus may have been more stable or more salient than temporal information, and that differ- 
ences in stability or salience produced the asymmetrical cross-dimensional interference observed in Experiments 1 and 2. One concern was that participants may have relied on spatial information to make temporal estimates because stimuli were situated in a constant spatial frame of reference (i.e., the computer monitor). For Experiment 3, stimuli were also situated in a constant temporal frame of reference. Temporal delay periods were introduced preceding and following line presentations, which were proportional to the spatial gaps between the ends of the stimulus lines and the edges of the monitor.

\subsection{Materials and procedure}

Nine participants from the MIT community performed Experiment 3, in exchange for payment. Stimulus materials and procedures were identical to those used in Experiment 2, with the following exception. In the previous experiments, the interval between the disappearance of the 'ready' screen and the appearance of the response prompt varied with stimulus duration. In the present experiment, this interval was fixed at $6400 \mathrm{~ms}$. Stimuli were preceded and followed by a delay period, which was proportional to spatial gap separating the ends of the line stimuli from the left and right edges of the monitor.

\subsection{Results and discussion}

The same pattern of cross-dimensional interference was found in Experiment 3 as in the previous experiments (cross-domain effects: Effect of displacement on duration estimation: $y=0.60 x+2604, r^{2}=.78, \mathrm{df}=7, p<.001$; effect of duration on displacement estimation: $y=0.0009 x+470, r^{2}=.03, \mathrm{df}=7, n s$. Within-domain effects: Effect of target displacement on estimated displacement: $y=0.80 x+73, r^{2}=.99$, $\mathrm{df}=7, p<.001$; effect of target duration on estimated duration: $y=0.68 x+866$, $\left.r^{2}=.99, \mathrm{df}=7, p<.001\right)$. The availability of a constant temporal frame of reference did not abolish the asymmetric influence of distance on time estimation (Fig. 2c).

\section{Experiment 4: Growing lines, concurrent tone}

Would space still influence participants' time estimates if stimulus duration were indexed by something non-spatial? For Experiment 4, a tone of constant frequency and amplitude accompanied each growing line. The tone began sounding when the line started to grow across the screen, and stopped sounding when the line disappeared. Thus, stimulus duration was made available to the participant in both the visual and auditory modalities, but stimulus displacement was only available visually.

\subsection{Materials and procedure}

Sixteen participants from the MIT community performed Experiment 4, in exchange for payment. Stimulus materials and procedures were identical to those 
used in Experiment 2, with the following addition. A constant tone $(260 \mathrm{~Hz})$ accompanied each growing line.

\subsection{Results and discussion}

Results of Experiment 4 replicated those of previous experiments (cross-domain effects: Effect of displacement on duration estimation: $y=0.55 x+2647, r^{2}=.90$, $\mathrm{df}=7, p<.001$; effect of duration on displacement estimation: $y=0.002 x+450$, $r^{2}=.10, \mathrm{df}=7, n s$. Within-domain effects: Effect of target displacement on estimated displacement: $y=0.72 x+96, r^{2}=.99, \mathrm{df}=7, p<.001$; effect of target duration on estimated duration: $\left.y=0.84 x+414, r^{2}=.99, \mathrm{df}=7, \quad p<.001\right)$. Displacement strongly influenced participants' duration estimates but not vice versa, even when temporal information was provided via a different sensory modality from the spatial information (Fig. 2d).

\section{Experiment 5: Moving dot}

Experiment 5 was designed to equate the mnemonic demands of the spatial and temporal dimensions of the stimulus. Rather than viewing a growing line, subjects viewed a dot that moved horizontally across the midline of the screen. In the previous experiments, just before each growing line disappeared participants could see its full spatial extent, from beginning to end, seemingly at a glance. By contrast, the spatial extent of a moving dot's path could never be seen all at once, rather it had to be imagined: Participants had to retrieve the dot's starting point from memory and compare it to the ending point in order to judge the distance that the dot traveled. The spatial and temporal dimensions of the dot stimulus had to be processed similarly in this regard: whenever we compute the extent of a temporal interval we must retrieve its starting point from memory.

\subsection{Materials and procedure}

Ten participants from the MIT community performed Experiment 5, in exchange for payment. Stimulus materials and procedures were identical to those used in Experiment 2, with one exception. Rather than viewing a growing line, subjects viewed a dot $(10 \times 10$ pixels $)$ that moved horizontally across the midline of the screen, from left to right.

\subsection{Results and discussion}

Results of Experiment 5 replicated those of previous experiments (cross-domain effects: Effect of displacement on duration estimation: $y=0.50 x+2452, r^{2}=.82$, $\mathrm{df}=7, p<.001$; effect of duration on displacement estimation: $y=-0.004 x+526$, $r^{2}=.29, \mathrm{df}=7, n s$. Within-domain effects: Effect of target displacement on estimated displacement: $y=0.92 x+55, r^{2}=.99, \mathrm{df}=7, p<.001$; effect of target duration on 
estimated duration: $\left.y=0.78 x+370, r^{2}=.99, \mathrm{df}=7, p<.001\right)$. As before, we found a strong and asymmetric cross-dimensional effect of space on time (Fig. 2e), suggesting that the perception of a long line was not necessary to lengthen participants' time judgments. Representations of spatial intervals that were never perceived at a glance but only reconstructed from memory were sufficient to modulate duration estimates.

\section{Experiment 6: Stationary lines}

Experiments 1-5 used moving stimuli. Is motion necessary to produce confusion between space and time, or would the asymmetric relationship between distance and duration still be found if static stimuli were used? In Experiment 6, participants viewed stationary lines and estimated either their displacement from end to end or the amount of time they remained on the screen, as in previous experiments.

\subsection{Materials and procedure}

Nineteen participants from the MIT community performed Experiment 6, in exchange for payment. Stimulus materials and procedures were identical to those used in Experiment 2, with the following exception. Rather than viewing growing lines, participants viewed stationary lines of various (spatial) lengths, which remained on the screen for various durations, according to the parameters used in Experiment 2.

\subsection{Results and discussion}

Results showed the same pattern of cross-dimensional interference found in all previous experiments (cross-domain effects: Effect of displacement on duration estimation: $y=0.28 x+2769, r^{2}=.72, \mathrm{df}=7, p<.002$; effect of duration on displacement estimation: $y=0.001 x+447, r^{2}=.10, \mathrm{df}=7, n s$. Within-domain effects: Effect of target displacement on estimated displacement: $y=0.84 x+32, r^{2}=.99$, $\mathrm{df}=7, p<.001$; effect of target duration on estimated duration: $y=0.83 x+423$, $r^{2}=.99$, df $\left.=7, p<.001\right)$. Duration estimates were strongly and asymmetrically dependent on the spatial length of the stimulus (Fig. 2f). This finding rules out the possibility that motion or speed was principally responsible for the results of the previous experiments.

An additional meta-analysis was conducted to evaluate cross-dimensional interference effects across all six experiments. A $2 \times 6$ mixed ANOVA with dimension (effect of distance on time estimation, effect of time on distance estimation) as a within-subjects factor and Experiment (Experiments 1-6) as a between-subjects factor compared the slopes of all cross-dimensional interference effects. Results showed a highly significant main effect of dimension, confirming that overall the slope of the effect of distance on time estimation $(M=0.51, S E=0.06)$ was greater than the slope of the effect of time on distance estimation $(M=0.001, S E=0.001$; $F(1,66)=83.73, \quad p<.0001)$. There was no main effect of Experiment $(F(5,66)=1.39, n s)$ and importantly, no dimension $\times$ experiment interaction 


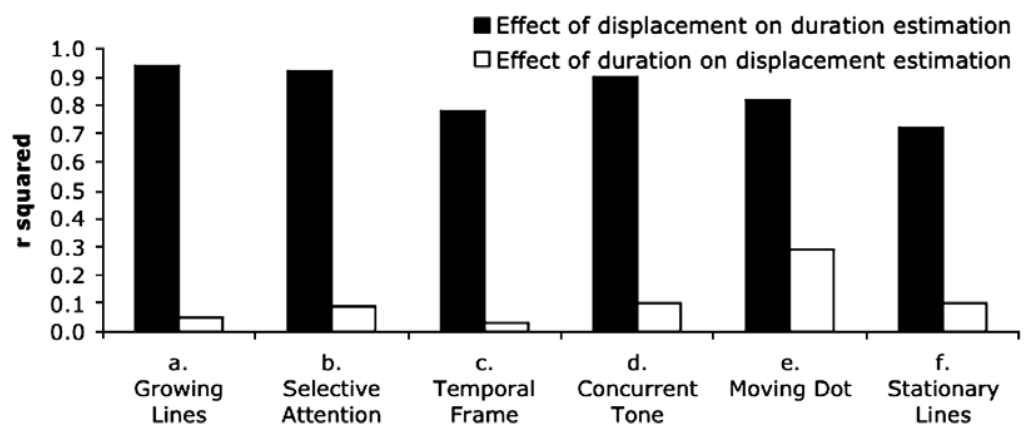

Fig. 2. Summary of cross-dimensional interference effects for Experiments 1-6. The effect of displacement on duration estimation was significantly greater than the effect of duration on displacement estimation for all experiments: (a) Growing lines: difference of correlations $=0.75 ; z=3.24, p<.001$. (b) Growing lines, selective attention: difference of correlations $=0.66 ; z=2.84, p<.002$. (c) Growing lines, temporal frame of reference: difference of correlations $=0.71 ; z=2.09, p<0.02$. (d) Growing lines, concurrent tone: difference of correlations $=0.63 ; z=2.60, p<0.005$. (e) Moving dot: difference of correlations $=1.45 ; z=3.69$, $p<0.001$. 2f, Stationary lines: difference of correlations $=0.54 ; z=1.62, p<0.05$. All $p$-values reflect onetailed $z$-tests.

$(F(5,66)=1.39, n s)$, indicating that the magnitude of the space-time asymmetry did not differ across experiments. To test for more subtle differences between experiments, one-way ANOVAs compared the slopes of different cross-dimensional interference effects, considered separately. No difference in slopes was found across experiments in the effect of distance on time estimation $(F(5,66)=1.40, n s)$ or the effect of time on distance estimation $(F(5,66)=0.57, n s)$. In summary, no significant differences were found in the pattern of cross-dimensional interference across experiments. The space-time asymmetry we report appears quite robust.

\section{General discussion}

When Piaget (1927/1969) investigated children's reasoning about space and time, he found that they often based their judgments of duration on their experience of distance. For example, when asked to judge the relative duration of two trains traveling along parallel tracks, children often reported (erroneously) that the train that traveled the longer distance took the longer time. Piaget concluded that children could not reliably distinguish the spatial and temporal components of events until about age nine. Like many contemporary results in cognitive science, our findings suggest that Piaget was right about the phenomenon he observed, but wrong about the age at which children resolve their confusion: apparently MIT undergraduates cannot reliably distinguish the spatial and temporal components of their experience, either.

There are, in principle, three possible relationships between people's mental representations of space and time. First, the two domains could be symmetrically dependent. John Locke (1689/1995) argued that space and time are mutually inextricable in our minds, concluding that "expansion and duration do mutually embrace and 
comprehend each other; every part of space being in every part of duration, and every part of duration in every part of expansion" (p. 140). Alternatively, our ideas of space and time could be independent. Any apparent relatedness could be due to structural similarities between essentially unrelated domains (Murphy, 1996, 1997). A third possibility is that time and space could be asymmetrically dependent. Representations in one domain could be parasitic on representations in the other (Boroditsky, 2000; Lakoff \& Johnson, 1980, 1999).

These three possible relationships predict three distinct patterns of cross-dimensional interference between space and time in the present experiments. If spatial and temporal representations are symmetrically dependent on one another, then any cross-dimensional interference should be approximately symmetric: distance should modulate duration estimates, and vice versa. Alternatively, if spatial and temporal representations are independent, there should be no significant cross-dimensional interference. However, if mental representations of time are asymmetrically dependent on mental representations of space as suggested by patterns in language, then we should observe an asymmetrical pattern in cross-dimensional interference: distance should affect duration estimates more than duration affects distance estimates. Results of all six experiments unequivocally support this third possibility, demonstrating that the asymmetric relationship between space and time found in linguistic metaphors is also found in our more basic non-linguistic representations of distance and duration.

Over the past century of psychophysical experimentation on space and time judgments, two effects have been demonstrated repeatedly: the Kappa effect and the Tau effect (Benussi, 1913; Bill \& Teft, 1969; Cohen, 1967; Cohen et al., 1954; Collyer, 1977; Helson, 1930; Jones \& Huang, 1982; Price-Williams, 1954; Sarrazin et al., 2004). In a typical experiment, three light bulbs were arranged in a row and flashed in succession, forming two spatiotemporal intervals. Participants were asked to compare either the spatial or temporal extents of the two intervals. Often, time judgments were found to increase as a function of the spatial separation between stimuli (the Kappa effect), and distance judgments were found to increase as a function of the temporal separation between stimuli (the Tau effect). At first glance, these experiments appear similar to those we report here; the Kappa effect seems consistent with our results, but the Tau effect appears inconsistent with our findings. Yet, our findings are easily reconciled with these classic findings, for two reasons. First, we hypothesize an asymmetric relationship between space and time (not a unidirectional relationship), our hypothesis can accommodate Tau-like effects of time on space judgments. Second, a survey of the literature suggests that Tau and Kappa effects emerge from implicit judgments of imputed velocity, and not from influences of the spatial or temporal components of the stimuli, per se (Jones \& Huang, 1982). We elaborate both of these points below.

\subsection{Asymmetrical vs. unidirectional effects}

The relationship between time and space in linguistic metaphors is asymmetrical, but not unidirectional. It is possible, in certain cases, to talk about space in terms of 
time. For example, we might say "we're only a few minutes from the subway" to indicate that the subway is a short distance away. Alternatively, however, this distance information could easily be conveyed using spatial language (e.g., we're only a few blocks from the subway). The space-time asymmetry in language is evident not only in terms of how frequently we talk about one domain in terms of the other, but also in terms of how obligatory these cross-domain mappings are. Whereas temporal metaphors for space are optional, spatial metaphors for time are so that pervasive they would be difficult for speakers to avoid using (Jackendoff, 1983; Pinker, 1997). Based on this asymmetry in language, we predicted asymmetrical crossdimensional interference between time and space in non-linguistic judgments. This prediction does not entail that time can never affect spatial judgments: rather, we predicted that for judgments on different dimensions of the same stimuli, the effect of space on time estimation should be greater than the effect of time on space estimation. We did not observe any significant effect of time on distance estimation, but such a finding would still be compatible with our hypothesis so long as we also found a significantly greater effect of distance on time estimation. We show such a significant difference between the cross-dimensional effects of space-on-time and time-on-space in all six experiments.

\subsection{The role of imputed velocity in Tau and Kappa effects}

A close examination of the literature reveals that the effects we report may be fundamentally different from Tau and Kappa effects. Although no theory on offer can fully explain Tau and Kappa effects (Sarrazin et al., 2004), the theory that appears to explain the majority of available data is the "imputed velocity hypothesis" (Jones \& Huang, 1982), according to which Tau and Kappa effects arise because "subjects impute uniform motion to discontinuous displays" (pp. 128; see also Anderson, 1974; Cohen, 1967; Collyer, 1977; Price-Williams, 1954). In most demonstrations of the Kappa effect (cf. Price-Williams, 1954) and in all known demonstrations of the Tau effect, participants judged the relative spatial or temporal extents of two or more successive intervals defined by discrete stimuli (e.g., spatiotemporally separated flashes of light). Although there was no actual or phenomenal motion in the stimuli, participants intuitively imputed motion at a given velocity to the flash of light as it 'traveled' from one bulb to the next. They produced errors when the imputed velocity of the stimulus changed between successive intervals, violating their intuition that it would continue to 'travel' between points with uniform velocity. Although experimenters explicitly manipulated the spatial and temporal extents of stimuli, the Tau and Kappa effects may be appropriately considered to be effects of imputed velocity on judgments of both time and space, rather than effects of time on space judgments or space on time judgments, per se.

Is it possible that participants imputed illusory velocity to our stimuli? This seems unlikely in Experiments 1-5 where the actual velocity was given by the stimuli, and even more unlikely in Experiment 6 in which there was no motion or speed information in stimuli at all - real or implied. Furthermore, there is 
no reason to believe that participants' expectations of constant velocity were violated, given that all moving stimuli moved at a constant velocity, all of our stimuli were spatiotemporally continuous, and none of our judgments required comparisons between successive intervals. Thus, our stimuli contained none of the 'active ingredients' of stimuli used to generate the Tau and Kappa effects. If imputing velocity to discontinuous successive intervals accounts for Tau effects, and our stimuli do not require participants to impute velocity or judge successive intervals, then we should not expect to find Tau-like effects (which, indeed, we do not). By the same token, we should not expect to find Kappa-like effects in our studies. The effects of distance on time estimation that we report are importantly different from imputed velocity-driven Kappa effects. Experiment 6 shows that the same asymmetric relationship of space on time is found even when static lines are used. This result converges with Cantor and Thomas's (1977) study showing that for very briefly presented static stimuli $(30-70 \mathrm{~ms})$, spatial information influenced temporal judgments but not vice versa (i.e., subjective duration increased as a function of stimulus area, but subjective area did not increase as a function of stimulus duration).

It is noteworthy that space influences temporal judgments even for the simple, brief temporal events we studied here which could in principle be mentally represented qua time, as proposed by interval-timer and accumulator models (Ivry \& Richardson, 2002). Thinking about time metaphorically in terms of space may allow us to go beyond these basic temporal representations. Mentally representing time as a linear path may enable us to conceptualize more abstract temporal events that we cannot experience directly through the senses (e.g., moving a meeting forward or pushing a deadline back), as well as temporal events that we can never experience at all (e.g., the remote past or the distant future). Metaphorical mappings from spatial paths, which can be traveled both forward and backward, may give rise to temporal constructs such as time-travel that only exist in our imagination.

\section{Conclusions}

Results of six experiments showed that mental representations of duration and displacement are asymmetrically dependent on one another. Judgments of temporal duration depended on information about spatial extent, but not the other way around. This pattern was predicted by the asymmetry of space-time metaphors in language. Although the brief durations used in these studies could in principle be mentally represented qua time, people still incorporated irrelevant spatial information into their temporal judgments. Moreover, these effects were obtained even in purely non-linguistic tasks: tasks that did not involve any linguistic stimuli or responses. These findings provide evidence that the metaphorical relationship between space and time observed in language also exists in our more basic representations of distance and duration, and suggest that our mental representations of things we can never see or touch may be built, in part, out of representations of physical experiences in perception and action. 


\section{Acknowledgments}

The authors thank Herb Clark, Steven Pinker, and the citizens of Cognation for discussion and comments on earlier drafts, and Shima Goswami, Jesse Greene, and Webb Phillips for programming and data collection. This research was supported by NSF CAREER Grant \#0608514 to L. Boroditsky, and by an NSF Graduate Research Fellowship and an NSF Dissertation Improvement Award to D. Casasanto. These studies constituted part of D. Casasanto's doctoral dissertation at MIT.

\section{References}

Alverson, H. (1994). Semantics and experience: Universal metaphors of time in english, mandarin, hindi, and sesotho. Baltimore: Johns Hopkins University Press.

Anderson, N. H (1974). Algebraic models in perception. In E. C. Carterette \& M. P. Friedman (Eds.). Handbook of perception (Vol. 2). New York: Academic Press.

Benussi, V. (1913). Psychologie der Zeitaufassung. Heidelberg: Carl Winter's Universitatsbuchhandlung.

Bill, J. C., \& Teft, L. W. (1969). Space-time relations: Effects of time on perceived visual extent. Journal of Experimental Psychology, 81(1), 196-199.

Boroditsky, L. (2000). Metaphoric structuring: Understanding time through spatial metaphors. Cognition, $75(1), 1-28$.

Boroditsky, L. (2001). Does language shape thought? Mandarin and english speakers' conceptions of time. Cognitive Psychology, 43(1), 1-22.

Boroditsky, L., \& Ramscar, M. (2002). The roles of body and mind in abstract thought. Psychological Science, 13(2), 185-189.

Bowerman, M. (1983). Hidden meanings: The role of covert conceptual structures in children's development of language. In D. R. Rogers \& J. A. Sloboda (Eds.), The acquisition of symbolic skills. New York: Plenum.

Cantor, N., \& Thomas, E. (1977). Control of attention in the processing of temporal and spatial information in complex visual patterns. Journal of Experimental Psychology: Human Perception and Performance, 3(2), 243-250.

Clark, H. H. (1973). Space, time, semantics and the child. In T. E. Moore (Ed.), Cognitive development and the acquisition of language (pp. 27-63). New York: Academic Press.

Cohen, J. (1967). Psychological time in health and disease. Springfield: Charles C. Thomas.

Cohen, J., Hansel, C. E. M., \& Sylvester, J. D. (1954). Interdependence of temporal and auditory judgments. Nature, 174, 642-644.

Collyer, C. E. (1977). Discrimination of spatial and temporal intervals defined by three light flashes: Effects of spacing on temporal judgments. Perception and Psychophysics, 21, 357-364.

Evans, V. (2004). The structure of time:Language,meaning and temporal cognition. Amsterdam: John Benjamins.

Gruber, J. (1965). Studies in lexical relations. Cambridge: MIT.

Helson, H. (1930). The tau effect - an example of psychological relativity. Science, 71(1847), 536-537.

Ivry, R., \& Richardson, T. (2002). Temporal control and coordination: The multiple timer model. Brain and Cognition, 48, 117-132.

Jackendoff, R. (1983). Semantics and cognition. Cambridge: MIT PRess.

Jones, B., \& Huang, Y. L. (1982). Space-time dependenacies in psychophysical judgment of extent and duration: Algebraic models of the tau and kappa effects. Psychological Bulletin, 91(1), 128-141.

Lakoff, G., \& Johnson, M. (1980). Metaphors we live by. Chicago: University of Chicago Press.

Lakoff, G., \& Johnson, M. (1999). Philosophy in the flesh: The embodied mind and its challenge to western thought. Chicago: University of Chicago Press.

Locke, J. (1689/1995). An essay concerning human understanding. Amherst: Promethius Books. 
Murphy, G. (1996). On metaphoric representation. Cognition, 60, 173-204.

Murphy, G. (1997). Reasons to doubt the present evidence for metaphoric representation. Cognition, 62, 99-108.

Núñez, R., \& Sweetser, E. (2006). Looking ahead to the past: Convergent evidence from aymara language and gesture in the crosslinguistic comparison of spatial construals of time. Cognitive Science, 30, 401-450.

Ornstein, R. (1969). On the experience of time. Hammondsworth: Penguin.

Piaget, J. (1927/1969). The child's conception of time. New York: Ballantine Books.

Pinker, S. (1989). Learnability and cognition: The acquisition of argument structure. Cambridge, MA, US: MIT Press.

Pinker, S. (1997). How the mind works. New York: Norton.

Price-Williams, D. R. (1954). The kappa effect. Nature, 173(4399), 363-364.

Sarrazin, J.-C., Giraudo, M.-D., Pailhous, J., \& Bootsma, R. J. (2004). Dynamics of balancing space and time in memory: Tau and kappa effects revisited. Journal of Experimental Psychology: Human Perception and Performance, 30(3), 411-430.

Sweetser, E. (1991). From etymology to pragmatics: Metaphorical and cultural aspects of semantic structure. Cambridge: Cambridge University Press.

Talmy, L. (1988). Force dynamics in language and cognition. Cognitive Science, 12, 49-100.

Torralbo, A., Santiago, J., \& Lupiáñez, J. (2006). Flexible conceptual projection of time onto spatial frames of reference. Cognitive Science, 30, 749-757.

Traugott, E. C. (1978). On the expression of spatiotemporal relations in language. In J. H. Greenberg (Ed.). Universals of human language: Word structure (Vol. 3, pp. 369-400). Stanford, CA: Stanford University Press.

Tversky, B., Kugelmass, S., \& Winter, A. (1991). Cross-cultural and developmental trends in graphic productions. Cognitive Psychology, 23, 515-557. 